OPEN ACCESS

Citation: Martone V. (2021) Abitare informale e regolazione violenta. Disagio abitativo e reti criminali ai margini della Capitale, in "Cambio. Rivista sulle trasformazioni sociali», Vol. 11, n. 21: 65-77. doi: 10.36253/cambio-10842

Copyright: (c) 2021 Martone V. This is an open access, peer-reviewed article published by Firenze University Press (http://www.fupress.com/cambio) and distributed under the terms of the Creative Commons Attribution License, which permits unrestricted use, distribution, and reproduction in any medium, provided the original author and source are credited.

Data Availability Statement: All relevant data are within the paper and its Supporting Information files.

Competing Interests: The Author(s) declare(s) no conflict of interest.
Monographic Section

\section{Abitare informale e regolazione violenta. Disagio abitativo e reti criminali ai margini della Capitale}

\author{
VitTorio Martone \\ Università di Torino \\ vittorio.martone@unito.it
}

\begin{abstract}
Informal housing includes the range of practices associated with producing or occupying residential spaces which falls beyond formal systems of urban planning. In this field it is complicated recognize what is legal or illegal and informal self-building and social innovation coexist with urban crime. On the one hand, informal housing is an opportunity for counter-movements against marketisation of the housing sector (squatting, self-construction, grassroots neighbourhood organisations). On the other hand, in the informal housing we can find urban crime, including organised crime groups, which control over the territory, occupy public housing, and manage public housing building. Massive urban security policies tackled informal space through segregation, surveillance, and punitive initiatives. Here the accusation of "mafia" has intensity the criminalisation of urban poverty, and the complexity of socio-spatial inequality is confused under the penal repression. The essay tries to deepen this ambivalence by looking at the outskirts of Rome and, in particular, at the relationship between informal housing in neighbourhoods with public housing complexes, with a high concentration of socio-economic disadvantage and high criminal density.
\end{abstract}

Keywords: informal housing, urban marginality, urban security policies, social inequality, organised crime.

La société libérale porte en soi le ghetto. La société de classes portait en soi le conflit et l'inégalité, mais pas le ghetto. Nous étions une société de discrimination, nous devenons une société de segregation (Touraine 1991: 12).

\section{ABITARE MARGINALE, ABITARE INFORMALE E ABITARE ILLEGALE: NOTA INTRODUTTIVA}

Nel rinnovato dibattito sui territori marginali, che ha riacceso l'attenzione tanto sulle aree interne e fragili, quanto sulle periferie urbane ${ }^{1}, l^{\prime}$ i-

\footnotetext{
${ }^{1}$ Mi riferisco ai «places left behind» (Wuthnow 2018), dove la distanza dalle appartenenze forti alimenta senso di abbandono (Rodriguez-Pose 2017) e forme di radicaliz-
} 
neguale accesso al diritto all'abitare compare come una delle più manifeste espressioni della disuguaglianza sociospaziale. La «nuova questione urbana», che ormai dagli anni Ottanta associa la marginalità residenziale alla marginalità sociale, assume nel tempo forme plurali e diversificate, che rimettono in discussione il concetto stesso di «periferie» (Petrillo 2013; Fregolent 2008), non più associabili ad aree geograficamente delimitate o separate dal «centro» (Laino 2020). La periferia nuova associa la disomogeneità sociale - con vecchie e nuove povertà, vecchie e nuove migrazioni - a una spiccata varietà spaziale, alternando vuoti abbandonati (aree dismesse, spazi interstiziali) o riempiti (aree commerciali, snodi autostradali, centri logistici di servizio alla città), con pieni "consolidati” (zone di costruzione storica, edilizia popolare pubblica, abusivo condonato) o di più recente auto-costruzione (informalità, occupazioni abitative, baraccopoli).

Riguardo a quest'ultimo aspetto, la proliferazione dell'abitare informale conferma una nota tendenza globale (Davis 2005) che non si ferma ai cosiddetti slums nelle metropoli del Sud del mondo, coinvolgendo le capitali europee $^{2}$ specie nella particolare declinazione dei modelli abitativi mediterranei ${ }^{3}$. Le solidarietà collettive e l'autoorganizzazione attorno all'abitare possono essere qui inquadrate come un policromo "contro-movimento" rispetto alla dismissione delle politiche di housing ${ }^{4}$, intese come l'implicazione più propriamente urbana del più generale disimpegno pubblico nel contrasto alle disuguaglianze $\mathrm{s}^{5}$. Ad esempio, la liberalizzazione del mercato degli affitti - a fronte di una crescente domanda inevasa di alloggi a prezzi accessibili - rinvigorisce diverse forme di autodifesa della società locale che proprio dall'informalità traggono alimento. Contro-movimento che ricomprende un insieme enorme di pratiche socio-spaziali che va necessariamente circoscritto. Mi riferirò in questa sede alla sfera del non pianificato, in cui si complica il confine tra legale e illegale. Più nello specifico, l'abitare informale risiede in quell'ampia area manipolatoria, che si insinua tra la «prassi» e la «norma» ${ }^{6}$, in cui rientrano tanto le forme di autorganizzazione collettiva e occupazione di edifici a scopo abitativo, quanto le forme di autocostruzione e abusivismo speculativo e privatistico. Un «quadro estremamente in disordine» (Paone et alii 2017: 9) che richiede una riduzione della scala di osservazione per tentare di indagare i motivi sociali, economici e politici di queste ampie trasformazioni dell'urbano che maturano al di fuori dei processi formali o pianificati.

A questo scopo, nelle pagine che seguono mi soffermerò sul caso di Roma, città mediterranea che esprime un coagulo esplicito di tutti questi aspetti. Più precisamente, guardando alle aree marginali della Capitale, mi concentrerò sul rapporto tra pratiche informali e complessi di edilizia pubblica a forte concentrazione di svantaggio sociale, in cui il costruito e l'auto-costruito si mescolano condizionandosi a vicenda nel dare forma allo spazio urbano. Qui terrò conto di due dimensioni: il ruolo della violenza che in queste aree può divenire criterio di regolazione sociale,

\footnotetext{
zazione politica ispirate all'anti-cosmopolitismo, all'autoritarismo e al nativismo (Norris e Inglehart 2019). In tal senso l'acuirsi delle disuguaglianze sociali rimette in discussione il delicato equilibrio tra capitalismi e democrazie e le leve dell'azione pubblica capaci di conciliare crescita e inclusione (Trigilia 2020).

${ }^{2} \mathrm{Si}$ pensi alle pratiche di encampement in cui si addensano migranti e profughi lungo i confini tra Paesi (Agier 2014).

${ }^{3}$ Caratterizzati da carenti politiche di edilizia pubblica, elevata diffusione della proprietà delle abitazioni e - di converso - ridotto numero di affitti, sia pubblici che privati (Allen 2006). Proprio alcune specificità riscontrabili nell'informalità urbana dei paesi mediterranei invitano a ridiscutere le dicotomie del tipo Nord/Sud del globo. Portando avanti queste argomentazioni, una recente modellizzazione dell'informalità abitativa in Italia mostra tre specificità prevalenti del fenomeno mediterraneo: coinvolge ampi gruppi sociali, non necessariamente svantaggiati; produce porzioni di tessuto urbano talvolta indistinguibili da quelle formalmente edificate; il mancato rispetto delle leggi sulla pianificazione rende l'informalità un fenomeno pervasivo, non eccezionale o marginale, sebbene non prevalente (Chiodelli et alii 2021).

${ }^{4}$ Il riferimento è alla categoria polanyiana, che indica reazioni sociali - non riconducibili a specifici interessi di classe - alle ondate di espansione delle logiche del capitale, come quelle registrate dopo la crisi finanziaria globale del 2008 che hanno indirizzato la governance pubblica verso una maggiore «protezione del mercato» rispetto alla «protezione della società» (Cfr. Palumbo e Scott 2019).

${ }^{5}$ Ricostruendo le trasformazioni del welfare state in un'ottica di growth regimes (il complesso delle strutture istituzionali, politiche e organizzative che presiedono al governo dell'economia), Hassel e Palier (2020) mostrano come proprio la riduzione delle politiche abitative e la finanziarizzazione del mercato immobiliare siano componente fondamentale tra i new engines of growth.

${ }^{6} \mathrm{~L}$ 'area manipolatoria indica qui i processi di riappropriazione, creativi e progettuali, in controtendenza rispetto ai processi di espropriazione della città (Cellamare 2008).
} 
intendendo le forme di violenza erogata in modo professionale e organizzato da reti e gruppi criminali ${ }^{7}$; le politiche di sicurezza che affrontano questa criminalità attraverso soluzioni puramente repressive, senza agire in altro modo sui contesti della povertà urbana e sul disagio abitativo ${ }^{8}$. Se, da un lato, queste politiche di contrasto registrano efficaci risultati nel contenere fenomeni criminali anche piuttosto capillari e violenti, dall'altro favoriscono interventi spesso centrati su controllo e militarizzazione - emergenziale, temporanea - dei territori, rischiando di criminalizzare i contesti nel loro insieme. A complicare il quadro capitolino è stato il massiccio ricorso alla strumentazione «antimafia», che nell'ultimo decennio ha contribuito all'incisività dell'azione penale a Roma e nel Lazio, ma ha anche catalizzato l'attenzione pubblica sulle «mafie», alimentata da media, cinematografia e letteratura militante, rendendo egemone un modello interpretativo eccezionalista e iperbolico che, «unito alla cartolina romanzo-criminalesca, ha mutato, in meridionalizzata e involuta, l'immagine di Roma nell'epoca di Mafia Capitale» (Meccia 2017: 198) ${ }^{9}$.

Uno scenario particolarmente calzante al caso di Ostia Lido, ampio agglomerato del X Municipio della Capitale che affaccia sul mare ${ }^{10}$. Con riferimento alle modalità di urbanizzazione descritte da Carlo Cellamare per tipizzare il «territorio abitato» romano, a Ostia sembrano palesarsi tanto le problematiche della periferia abusiva, quanto quelle del quartiere di edilizia residenziale pubblica, in cui

la malavita organizzata si inserisce tra l'onestà e la povertà [...]. Quartieri "pubblici“ dove il pubblico non è presente: ne sono un esempio evidente la diffusione del mercato informale della casa e delle occupazioni ad esso legate. Per tutti questi motivi sono spesso oggetto di stigmatizzazione che ne incoraggia fortemente la ghettizzazione, anche nell'immaginario collettivo, oltre a subire la facile strumentalizzazione da parte dei mass media (Cellamare 2016: 169-170).

Come in altre periferie romane, a Ostia si registrano fenomeni di elevata concentrazione di svantaggio sociale e residenziale con quartieri malmessi in cui la questione abitativa finisce per addensare in spazi circoscritti diverse forme di potenziale marginalità strutturale (disagio abitativo, insufficienza dei servizi pubblici) e socio-economica (deprivazione, dispersione scolastica e svantaggio educativo, segregazione). Questi quartieri sono sede di attivazione e riattivazione per molteplici ed eterogenee esperienze che negli anni alimentano reti sociali e appartenenza. A un tempo, sacche di deprivazione spazialmente circoscritte e assenza del pubblico possono facilitare una certa malavita di strada, capace di alimentare maestranze anche di gruppi organizzati. Entrambi i fenomeni coabitano nell' informalità ai margini, talvolta contendendosi il presidio degli spazi, più spesso ignorandosi. Scopo del saggio è tentare di discernere i fenomeni sociali da quelli criminali, concentrandosi su questi ultimi e situandone l'operatività proprio all'interno di ampie sacche di informalità.

Il saggio si suddivide in quattro paragrafi. Nel secondo preciso ulteriormente le categorie analitiche e le ipotesi di partenza, declinandole all'interno della questione sociale e abitativa romana, nel cui quadro si inscrive la genesi delle forme criminali autoctone e delle politiche di sicurezza implementate. Nel terzo paragrafo ricostruisco l'evoluzione urbanistica e demografica di Ostia Lido, che vede la configurazione di quartieri a forte criticità strutturale e

\footnotetext{
${ }^{7} \mathrm{Nei}$ contesti connotati dalla pervasività di pratiche informali o irregolari può registrarsi una certa domanda di regolazione, acuita dall'assenza del soggetto pubblico, in cui trovano spazio attori in grado di usare violenza professionale e organizzata. Con riferimento alla criminalità "mafiosa", proprio nell'area grigia al confine fra legale e illegale la violenza - materiale e simbolica - è veicolo di arricchimento e di controllo del territorio e, a un tempo, di accumulazione di risorse relazionali, sociali e culturali, importanti per costruire consenso, reputazione e legittimazione (Sciarrone 2019a).

${ }^{8} \mathrm{Mi}$ riferisco agli orientamenti di policy che, nella complessiva colpevolizzazione e segregazione della povertà (Wacquant 2009), riconducono all'etichetta «criminale» lo spettro dei comportamenti indecorosi (Pitch 2013) o antagonisti (Selmini 2020), considerati disordine urbano (Paone 2012). Applicata dagli anni Novanta alle periferie sensibili, «a rischio» o «pericolose», questa impostazione ha nel tempo favorito soluzioni di tipo securitario e punitivo, disperdendo la complessità dei margini.

${ }^{9} \mathrm{Al}$ di là delle fiction, solo per richiamarne alcuni titoli, si va da Roma mafiosa (Capaldo 2013) a Roma brucia (Orsatti 2015), da Capitale infetta (Sabella 2016) a 2000 anni di corruzione (Angeli 2020). I documentari televisivi non escono dal canovaccio, come Roma criminale di La7 e Ostia Criminale - La mafia a Roma di Tv8.

${ }^{10}$ Il Comune di Roma si compone di 15 Municipi. Il X, oltre a Ostia Lido (Nord e Sud), comprende i settori di Acilia (Nord e Sud), Casal Palocco, Castel Fusano, Castel Porziano, Infernetto, Malafede e Ostia Antica. Secondo i dati Istat (dicembre 2019), nel X Municipio abitano 231.220 persone, di cui 79.901 a Ostia Lido, agglomerato compatto e densamente popolato adiacente al litorale.
} 
sociale. Anche la separazione dal resto della città e le forme dell'edilizia pubblica locale danno forma alle presenze criminali locali, che usano occupazioni e gestione di alloggi popolari come forma di presidio territoriale. Nell'ultimo paragrafo, tirando le somme, volgo lo sguardo all'azione repressiva recente e al dibattito pubblico locale che, concentrato sull'antimafia penale, rischia di trascurare l'attenzione sui contesti.

Impianto teorico e parte del materiale empirico traggono spunto da ricerche condotte a partire dal 2012 sui processi di genesi e di riproduzione di fenomeni classificati come «mafiosi» a Roma e nel Lazio ${ }^{11}$. Dialogando con letterature storico-sociali in tema di sviluppo locale, studi urbani, political economy e sociologia della criminalità organizzata, si è tentato di inquadrare i gruppi criminali in contesti analizzati nella loro dimensione spaziale (in senso territorialista, con riferimento ai profili demografici, urbanistici, ecologici ecc.), socio-economica (processi di regolazione pubblica dei mercati, sacche di informalità o illegalità diffusa connesse alla struttura o alla marginalità sociali) e politico-istituzionale (assetti organizzativi del governo locale e dinamiche di legittimazione e costruzione del consenso su scala territoriale). La stesura di questo saggio si arricchisce di nuove acquisizioni bibliografiche ed empiriche recenti, specialmente in riferimento alla chiusura del procedimento di secondo grado che ha confermato l'associazione a delinquere di stampo mafioso per uno dei gruppi criminali di Ostia (gennaio 2021). Oltre all'analisi della letteratura e del materiale di inchiesta ${ }^{12}$, è stata cruciale un'approfondita osservazione diretta, arricchita da conversazioni libere e interviste semi-strutturate ${ }^{13}$, dalla rassegna della cronaca locale e dall'esame dei materiali di denuncia prodotti all'antimafia civile.

\section{PERIFERIE, DISAGIO ABITATIVO E POLITICHE DI SICUREZZA: LE «MAFIE DI MEZZO»}

La storica debolezza del governo capitolino nell'amministrare le trasformazioni urbane è stata efficacemente interpretata nel «regime dell'Urbe», in cui la centralità dei costruttori e della rendita fondiaria e immobiliare è tratto distintivo (D’Albergo e Moini 2015: 12). Il regime pare avere proprio nell'informale una logica di fondo, incastrandosi in un quadro di reciprocità opache tra pubblico e privato, dove la politica ha il compito ancillare di riprodurre le condizioni necessarie a garantire il perpetuarsi delle rendite romane (Benini e De Nardis 2013). Dalla metà degli anni Novanta, in piena Tangentopoli, Roma avvia un processo di «neo-liberalizzazione dell'azione pubblica» (D'Albergo e Moini 2015: 59) in cui la massiccia dismissione dell'intervento pubblico viene presentata anche come strategia di contrasto alla pervasività della corruzione che inficia l'amministrazione capitolina. Privatizzazione, managerializzazione e competizione internazionale sono alla base del programma di governo che, tuttavia, in fase di implementazione, non sembra arginare l'opacità del regime urbano, creando invece una «perfetta fusione delle esigenze neo-liberali con quelle clientelari» (Violante e Vicari 2018: 169).

Sul fronte dell'abitare, regolazioni particolaristiche avvantaggiano interessi al confine fra privatismo e illegalità (De Leo 2016) in un «assemblage peculiare di risorse cognitive, organizzative, istituzionali ed economiche» (Coppola 2016: 224): si va dall'auto-costruzione abusiva d'immobili all'appropriazione indebita di alloggi popo-

\footnotetext{
${ }^{11}$ L'attività è iniziata con un progetto di ricerca coordinato da Rocco Sciarrone nell'ambito del «Laboratorio di Analisi e Ricerca sulla Criminalità Organizzata» dell'Università di Torino finanziato dalla Fondazione Res di Palermo, i cui risultati sono pubblicati in Mafie del Nord. Strategie criminali e contesti locali (2014, ristampato in forma aggiornata nel 2019). Un approfondimento monografico su tre contesti specifici (il Lazio meridionale, il litorale romano e l'area metropolitana romana) è in Martone (2017).

${ }^{12}$ Prevalentemente Ordinanze e Sentenze della Dda di Roma, relazioni della Direzione Nazionale Antimafia (Dna), della Direzione Investigativa Antimafia e resoconti della Commissione Parlamentare Antimafia. A questo materiale si aggiungono i dati raccolti nel prezioso rapporto annuale Mafie nel Lazio, curato dall'Osservatorio Tecnico Scientifico per la Sicurezza e la Legalità della Regione Lazio e giunto alla $\mathrm{V}$ edizione (2020).

${ }^{13} \mathrm{Nel}$ complesso ho somministrato 51 interviste semi-strutturate ad abitanti, operatori della cooperazione sociale, imprenditori, attivisti antimafia, giornalisti, sindacalisti, amministratori locali, magistrati e studiosi del fenomeno. Fino al 2018 ho svolto una costante attività di campo, specie nei periodi di intensificazione del dibattito o dell'azione penale, in cui ho raccolto testimonianze in conversazioni libere, partecipazione a dibattiti pubblici, eventi promossi dall'antimafia giudiziaria e civile, manifestazioni politiche e assemblee pubbliche.
} 
lari; dalla gestione privatistica dell'auto-recupero al massiccio ricorso al condono edilizio ${ }^{14}$. L'opacità non connota solo il mercato abitativo. Nell'area metropolitana romana si registra «una vorace ed intensa criminalità economica, rappresentata dalle grandi bancarotte, dalle maxi evasioni fiscali o dalle clamorose truffe in danno dello Stato o di enti pubblici» (Dna 2016: 912) inserita in un contesto di illegalità diffusa. Economia non osservata, evasione fiscale e irregolarità contrattuale e contributiva risultano endemiche in settori chiave della crescita economica e demografica della Capitale successiva alla crisi finanziaria del 2008, crescita comparativamente superiore alle altre realtà metropolitane del Paese (Casucci e Leon 2014). Soprattutto il commercio al dettaglio e i servizi tradizionali sostenuti dalla turistificazione incontrollata (ristorazione, facchinaggio, pulizia, trasporto, sicurezza privata) creano un'ampia domanda di lavoro in parte coperto da manodopera dequalificata e impiegata anche in forme irregolari o grigie. Una «tumultuosa espansione demografica, che, fatte le dovute proporzioni, presenta alcune analogie con quanto accaduto nel corso degli anni cinquanta e sessanta» (Carminucci et alii 2014: 100). Come noto, il boom economico del Dopoguerra aveva dato alla Capitale la connotazione di città-regione, producendo «il ridimensionamento della classe contadina» e attraendo consistenti flussi migratori (Cassetti 2008: 25). L'edificazione di quartieri dormitorio non era bastata a governare i baraccamenti e lo spontaneismo abitativo diffuso, che rispondono a un'esigenza abitativa, ma aggravano gli squilibri socio-spaziali preesistenti ${ }^{15}$. L'espansione romana recente sembra chiudere il cerchio: le dinamiche demografiche e urbane si dipanano ancora nel connubio tra questione abitativa e speculazione edilizia sui valori immobiliari che aumenta ulteriormente il divario tra centro e periferie, con processi di marginalizzazione e concentrazione dello svantaggio (Lelo et alii 2019), disorganizzazione sociale e radicalizzazione politica (Bertuzzi et alii 2019). In questi ambiti si palesa un uso politico dell'informalità urbana che, da un lato, criminalizza in maniera mirata solo le pratiche dei gruppi marginali e, dall'altro, prosegue nell'affrontare i problemi sociali in linea emergenziale, attraverso sgomberi e trasferimenti (Chiodelli et alii 2021). Ne conseguono periferie differenziate e plurali, dove si addensano vecchie e nuove forme di deprivazione, luoghi di accoglienza informale ${ }^{16}$ talvolta oggetto di contesa "etnica"17 e occupazioni promosse dai movimenti di lotta ${ }^{18}$. Neanche il patrimonio di edilizia pubblica rappresenta un argine all'ineguale accesso alla casa. Non solo per la sua dimensione - rappresenta comunque un terzo del mercato degli affitti in città ${ }^{19}$ - quanto per l'opacità della sua gestione. La «scarsa mobilità del bene, dovuta in parte alle leggi che lo regolano, in parte alla carenza di controlli e in parte alle prassi di chi lo abita, ne inficiano la validità tanto da renderlo un bene congelato» (Puccini 2018: 279). Metà degli alloggi si concentra nelle aree est e sud della Capitale, come nei Municipi VI (Tor Bella Monaca, Torre Maura, Torre Spaccata e Ponte di Nona) e IV (San Basilio e Tiburtino), poi nel III (Tufello e Monte Sacro) e nel Municipio $\mathrm{X}$, con le già citate Acilia e Ostia Lido.

\footnotetext{
${ }^{14}$ Nell'analizzare la "politica dell'abusivismo" a Roma, Alessandro Coppola scrive: «Con oltre 670.000 richieste presentate fra il 1985 e il 2004, ovvero poco meno di una ogni quattro residenti, quello di Roma è largamente il comune con la quantità più elevata di domande di condono» (2018: 120).

${ }^{15} \mathrm{Si}$ tratta delle celeberrime borgate, «acquartieramenti di povera gente appartenente tutte allo stesso ceto» in agglomerati intermedi tra città e campagna, esito del «piccone demolitore» fascista (Insolera 2011: 135-145). Sono inaugurate dal 1924, in occasione della costruzione di Acilia, inclusa nel territorio del X Municipio, oggetto del prossimo paragrafo.

${ }^{16}$ Ad esempio, nel 2004 viene occupato l'ex Hotel Africa, un magazzino ferroviario dismesso che poi accoglierà centinaia di richiedenti asilo. Dallo sgombero dell'ex Hotel nasce l'occupazione di Palazzo Selam, stabile di nove piani abitato in condizioni di grave precarietà (Cittadini del Mondo 2018).

${ }^{17}$ Nelle periferie si concentrano insediamenti informali, centri di accoglienza e campi rom, anch'essi in un quadro di disordine istituzionale. Qui la vivace propaganda di estrema destra - quasi sempre promossa da Casapound - fomenta proteste contro l'apertura di centri di accoglienza (come a Tor Sapienza, nel 2014), l'assegnazione di alloggi popolari agli stranieri (come a San Basilio nel 2016 e a Casal Bruciato nel 2019), lo sgombero di famiglie rom (Torre Maura, 2019), tutte sostenute da slogan razzisti del tipo «Stop invasione», «Basta immigrati incivili», «Via i rom, ridateci gli africani».

${ }^{18}$ Per dare un ordine di grandezza, la Commissione parlamentare di inchiesta sulle condizioni di sicurezza e sullo stato di degrado delle città e delle loro periferie parlava di 99 occupazioni a scopo abitativo, di cui 79 in zona suburbana (2017). Sono disponibili ricerche sul campo su circa 15 immobili occupati per un totale di circa 15.000 persone (Grazioli e Caciagli 2017).

${ }^{19} \mathrm{Il}$ patrimonio abitativo romano conta 74.000 alloggi popolari, pari al 7\% dello stock immobiliare, ma che tuttavia coprono ben il 46,8\% del mercato degli affitti (Puccini 2016).
} 
Negli anni Novanta, nel pieno del suddetto processo di neo-liberalizzazione dell'azione pubblica, queste e altre periferie sono divenute «quartieri sensibili» (Farruggia e Ricotta 2010), sede di sperimentazione locale della nuova stagione di politiche di sicurezza urbana, con approcci di prevenzione situazionale dagli esiti piuttosto limitati (Battistelli e Lucianetti 2010). Proprio in questi quartieri popolari opera oggi un ginepraio di gruppi criminali che esprime le più spiccate forme di presidio territoriale, le cui attività ben si adattano alle configurazioni socio-spaziali strutturatesi negli anni. Dal traffico di stupefacenti - che assume la forma delle "piazze di spaccio" - allo sfruttamento della prostituzione, dal gioco illegale all'usura e alle estorsioni, fino al racket sulle case popolari e all'occupazione abusiva di alloggi. Ad esempio, tra Tor Bella Monaca e San Basilio la criminalità si atteggia «secondo il modello delle "piazze di spaccio" importato dal territorio campano» (Dna 2016: 917) che si estende «con modalità "industriali" » in diverse periferie (Dna 2012: 719), attraverso zone controllate da vedette, distribuzione parcellizzata e depositi temporanei per gli stupefacenti. Il racket sulle case e l'occupazione di alloggi popolari servono a perimetrare in maniera tangibile il territorio controllato e ad allocarvi famiglie vicine, in una sorta di welfare criminale offerto anche in cambio della periodica custodia temporanea di stupefacenti ${ }^{20}$. Presidio territoriale e costruzione del consenso rendono intelligibili anche i non rari casi di aggressione e ribellione diffusa agli interventi delle forze di polizia, fattisi progressivamente più insistenti non solo nel contrasto alle occupazioni abusive, ma soprattutto al traffico di stupefacenti e alle associazioni criminali radicate nei quartieri di edilizia pubblica. Tra il 2011 e il 2018 le operazioni antidroga a Roma sono cresciute di un terzo (da 2.862 a 3.703, $+29,3 \%$ ), le persone denunciate di un quinto (da 4.219 a $5.042,+19,5 \%$ ), ma soprattutto sono cresciuti significativamente gli arresti per «associazione» finalizzata al traffico di stupefacenti $(\text { dal } 7,5 \text { al 24,2\%, }+16,7 \%)^{21}$. Sottolineo il ricorso alla contestazione dei reati associativi perché segnano un cambio di passo della locale Direzione Distrettuale Antimafia che, proprio dal 2012, avvia diverse operazioni che porteranno alla disarticolazione dei gruppi criminali autoctoni, cui viene sempre più spesso contestata anche la natura "mafiosa" 22 .

Su questo punto è bene soffermare l'attenzione. La strumentazione antimafia è apparsa più pervasiva ed efficace nel contenimento dell'ala militare dei sodalizi e delle loro più immediate adiacenze, ma è stata anche significativamente potente nelle dinamiche di etichettamento delle periferie. Qualificare come "mafia" i gruppi criminali nei quartieri di edilizia popolare ha assunto una funzione fortemente performativa, mutandone non solo la consistenza penale, ma anche quella politica e pubblica, estendendo l'aggettivazione "mafiosa" all'intera città (cfr. ad esempio Capaldo 2013; Savatteri e Grignetti 2015; Orsatti 2016) e travolgendo anche il dibattito sulle periferie (cfr. ad esempio Bulfon 2019; Trocchia 2019). Quando assume toni spazialmente connotati, infatti, l'aggettivazione mafiosa può alimentare anche una precisa costruzione politica del «male» urbano ${ }^{23}$, incarnato in quartieri «pericolosi», luoghi riconoscibili, mondi paralleli, che favorisce reazioni centrate su controllo, militarizzazione e ulteriore segregazione di gruppi svantaggiati, migranti o dissidenti. Ne sono un esempio la stigmatizzazione (Vereni 2015) e poi la repressione dei movimenti per il diritto all'abitare, con il massiccio ricorso alle demolizioni e agli sgomberi delle occupazioni a scopo abitativo (Caciagli 2018). Informalità e irregolarità diffusa sono dunque un contesto di azione deprivato, ma anche un potenziale per chi occupa immobili per sopperire all'assenza del pubblico, agendo nella

\footnotetext{
${ }^{20}$ Nell'estate 2015 l'amministrazione capitolina avvia accertamenti sul patrimonio comunale e dell'Erp (Edilizia residenziale pubblica) rilevando 743 appartamenti occupati irregolarmente, dei quali circa 50 attribuibili ad affiliati a una nota rete criminale romana di origine sinti. Lo stesso modello è stato individuato all'interno del cosiddetto "Casermone", complesso di edilizia popolare di Frosinone.

${ }^{21}$ Fonte: Ministero dell’ Interno - Dipartimento della Pubblica Sicurezza (Direzione Centrale dei Servizi Antidroga).

${ }^{22}$ Dal 2012 la Procura di Roma ha cambiato guida e i nuovi procuratori propongono «un "cambio di metodo" [...] che riunisce in sé un'idea di mafia (e di antimafia) e l'articolazione di un metodo investigativo» (Ciccarello 2016: 72). Sul punto si è aperto un acceso dibattito sull'applicabilità della normativa antimafia in aree di insediamento non tradizionale (cfr. fra gli altri Visconti 2015; Pignatone e Prestipino 2019; Sciarrone 2019c).

${ }^{23}$ Traggo ispirazione dal lavoro di Francesco Benigno (2015) sull'uso della paura politica come strumento di governo: se il discorso sulla sicurezza urbana può essere letto come una forma di costruzione sociale e politica del «male» incarnato da «classi pericolose» in grado di rappresentarlo, i paesaggi urbani «degradati» e «insicuri» risultano una forma di spazializzazione del male in luoghi in grado di rappresentarlo.
} 
sfera del non pianificato e connotando una perifericità progettuale, fulcro di autorganizzazione comunitaria (Cellamare 2019). Anche i gruppi criminali si esprimono in questa sfera dell'abitare informale, ma i due fenomeni non possono essere confusi, tantomeno possono essere affrontati con gli stessi strumenti analitici (e repressivi).

Provo a entrare più nello specifico di queste considerazioni attraverso il caso di Ostia Lido, tentando di situare i fenomeni criminali nel processo storico di trasformazione di una periferia.

\section{PRATICHE INFORMALI, COMPLESSI DI EDILIZIA PUBBLICA E REGOLAZIONE VIOLENTA}

Sebbene le bonifiche iniziali del litorale comincino alla fine del XIX secolo ${ }^{24}$, la prima organica proposta di urbanizzazione per Ostia si può far risalire al progetto SMIR ${ }^{25}$, dal nome dell'ente che nel 1920 ottiene un finanziamento pubblico per la formazione, verso il mare, di un grande quartiere industriale. Un proposito abbandonato solo tre anni dopo, quando le redini dell'urbanistica della Capitale passano al governatorato fascista. In effetti, i successivi diciotto anni di urbanistica capitolina saranno influenzati dalla volontà del Regime di ampliare Roma verso il mare; solo per citare alcune opere: nel 1923 viene approvato il piano per Ostia e un anno dopo è inaugurata la Ferrovia Roma-Lido. Come accennato, il 1924 segna l'avvio dell'edificazione di Acilia, la prima «borgata». L'avanzare degli interventi per Ostia, come l'inaugurazione dell'Idroscalo alla foce del Tevere (1925) e la rifinitura del Lungomare Toscanelli (1933) appaiono come «piccole iniziative slegate e senza alcun nesso con un'eventuale espansione di Roma» (Insolera 2011: 134). Nonostante i propositi del Regime, infatti, anche il progetto dell'esposizione universale del 1942 non andrà oltre l'attuale quartiere Eur. Ai fini della trattazione che segue, questi elementi sono di interesse perché imprimono una relativa separazione spaziale e urbanistica del quartiere, un'indole di città autonoma che nel tempo diverrà oggetto di tematizzazione politica anche attraverso richieste di maggiore autonomia ${ }^{26}$.

Nel secondo dopoguerra il litorale viene investito dall'espansione galoppante della Capitale, cedendo il passo «all'edilizia speculativa che, complice il disinteresse delle istituzioni, consentì di agire con interventi di sostituzione degli edifici storici e di manipolazione di quelli rimasti, omologando la cittadina costiera [...] alle periferie - pianificate o abusive - della Capitale» (Creti 2008: 26). Ostia comincia così a configurarsi come quartiere periferico di Roma salendo ai primi posti nella classifica dei territori con maggior numero di abusi edilizi ${ }^{27}$. Agli inizi degli anni Settanta prende avvio il trasferimento dei cosiddetti baraccati nel quartiere di Nuova Ostia, esito di una fallimentare speculazione residenziale privata, convertita dal Comune di Roma a quartiere di edilizia pubblica, con l'acquisto e l'affitto delle note «palazzine Armellini». Il primo popolamento di Nuova Ostia si caratterizza per un intenso fervore dei comitati di lotta per la casa. Gli alloggi sono in gran parte occupati prima delle assegnazioni e i nuovi residenti si organizzano in diverse forme associative per rivendicare manutenzione e forniture di base. Come mi racconta un abitante:

\footnotetext{
${ }^{24}$ Il primo progetto di bonifica delle paludi salmastre che connotano la foce del Tevere (Ostia e Maccarese) viene approvato nel 1880 e portato avanti dal 1887 da una colonia di lavoratori ravennati, fino al 1908. Poco più tardi (1916), viene presentato il «Piano Regolatore di Ostia Nuova» improntato al modello di città-giardino che ispira l'urbanistica dell'epoca: edifici bassi e spazi verdi, destinati essenzialmente alla funzione di villeggiatura.

${ }^{25}$ Ente per lo sviluppo marittimo e industriale di Roma.

${ }^{26}$ Almeno tre proposte di legge di iniziativa popolare hanno richiesto la secessione amministrativa dalla Capitale (1989, 1999 e 2016). Va poi sottolineato che il Municipio gode negli anni di forme più accentuate di autonomia rispetto agli altri governi subcomunali della Capitale. Dal 1992 esiste un «Regolamento speciale del decentramento» (Deliberazione del Consiglio Comunale n. 281/1992). Si tratta della cd. «delibera Pannella», con riferimento a Marco Pannella, all'epoca nominato Presidente del Municipio (allora Circoscrizione XIII) in seguito al commissariamento imposto dal Prefetto di Roma per gravi casi di corruzione. Il Regolamento speciale delega la competenza sulle spiagge, escludendo solo la Riserva naturale del Litorale Romano, a sud di Ostia. Nel 2011 l'amministrazione Alemanno concede ulteriore autonomia specialmente sull'importante demanio marittimo (Deliberazione del Consiglio Comunale n. 18/2011).

${ }^{27}$ Il più noto è nell'area dell'Idroscalo, che da almeno settant'anni accoglie diverse ondate migratorie sperimentando il susseguirsi di insediamenti spontanei e di baraccamenti precariamente delimitati tra il Tevere e il litorale. Qui l'autocostruzione prosegue da decenni tra fasi di tolleranza e fasi di demolizione, a seconda delle tensioni politiche (cfr. Portelli 2018).
} 
Per organizzare la nostra spinta fu fatto una sorta di Comitato di lotta per la casa di Ostia, in cui il Pci mise tutte le forze dell'arco costituzionale. Costituivamo Comitati di Caseggiato. Arrivammo fino alla auto-riduzione delle bollette della luce. Stiamo parlando degli anni Settanta. Quell'ondata comportò due grossi effetti negativi. Trasferimento di tutte quante le borgate a Nuova Ostia ... questo mischiare, mischiare ... specialmente nell'ultima ondata, recava in sé manifestazioni di criminalità organizzata, con collegamenti di manovalanza della Banda della Magliana. L'altra cosa che noi evidenziammo subito, dopo due anni, era che soprattutto le persone che arrivavano dai borghetti di Roma rivendevano casa [...]. A te, della borgata pinco pallino, t'assegnano la casa del comune e ti trasferiscono a Ostia. Tu che fai? La prendi. Ma, se la tua economia vitale sta di là, da operaio o artigiano, è chiaro che alla lunga non ce la fai. Anche perché Nuova Ostia era isolata. Poi sapevamo come erano state costruite queste cacchio di case. Con la sabbia di mare, col cemento armato fittizio, piene di problemi. Da qui iniziò una roba che secondo me ancora sussiste. Una volta che c'era la sanatoria del borghetto, avviato questo, nel vuoto della riprogettazione urbanistica su quel territorio, cominciavano a risorgere altre baracche. La baraccopoli che genera altre baraccopoli (Int. referente comitato locale).

Diversi interventi simili trasformano progressivamente il litorale in valvola di sfogo per le eccedenze della città. Queste trasformazioni sono esito e causa di due fenomeni, strettamente interconnessi: da un lato, l'incancrenirsi di relazioni collusive nei circuiti politico-amministrativi, poggiati sulla complicità di dirigenti, funzionari pubblici, costruttori e politici, facilita la proliferazione di illegalità diffuse nel consumo di suolo. Dall'altro, il «boom edilizio» trasforma il litorale in periferia, accogliendo una parte rilevante della popolazione romana e di nuova immigrazione che non trova collocazione nel perimetro della città ${ }^{28}$.

In questo scenario, secondo le diverse fonti giudiziarie, questi stessi quartieri esprimeranno nel tempo le più articolate reti di narcotraffico romane, riconvertendo soprattutto la zona di piazza Gasparri, a Nuova Ostia, in una delle più importanti "piazze di spaccio" della Capitale. Sul litorale operano gruppi criminali con una forte impronta familiare, tradizionalmente dediti - oltre agli stupefacenti, all'usura e alle estorsioni - all'occupazione e al controllo degli alloggi popolari ${ }^{29}$. L'occupazione avviene con l'uso della violenza esplicita e l'intimidazione utili a manutenere un presidio territoriale anche circoscritto a pochi isolati, ma esercitato con forme di prevaricazione piuttosto esplicite. Il loro imporre lo sgombero degli alloggi rientra in una più ampia pratica di perimetrazione tangibile del territorio presidiato, espresso anzitutto nei confronti dei rivali, come racconta una testimone:

avevano condotto con la forza mio marito nei pressi di piazza Gasparri e qui lo avevano selvaggiamente picchiato prendendolo anche a morsi [...]. Nell'arco degli ultimi 6 o 7 mesi mio marito è stato prelevato e picchiato almeno 10 volte. [...]. Più volte [...] sono entrati nella casa in cui mi trovo [...]. Nel corso di ulteriori visite intimidatorie. [Un esattore, $n d r$ ], sieropositivo, ha minacciato me e mio marito con un'arma artigianale e affilata dicendo che ci avrebbe infilzati tutti e infettati contagiandoci con l'Hiv (Tribunale di Roma 2018: $137-8)^{30}$.

Il racconto di una collaboratrice, molestata continuamente da uno degli affiliati, che la spinge a prostituirsi in cambio dell'alloggio in cui vive («non perdeva occasione per avvicinarmi e toccarmi nelle parti intime aggiungendo che sarebbe stato meglio se io avessi fatto la zoccola per lui»), conferma il quadro di violenza diffusa.

$\mathrm{Su}$ questo punto non si può non richiamare lo stato di abbandono dell'edilizia residenziale pubblica ricostruito in apertura, in cui proliferano - sin dalla sua prima edificazione - occupazioni, abusivismi e contenziosi amministrativi $^{31}$. Ancora una testimonianza ci immerge nella lotta quotidiana per l'ottenimento di una casa, praticata con sistematica prepotenza, plausibile solo se calata nel contesto di profondo degrado in cui le parti convivono:

\footnotetext{
${ }^{28}$ Dal 1971 al 2015 la popolazione del Municipio è più che raddoppiata - 99.917 a 230.544 abitanti (+230,7\%), senza un adeguamento e un miglioramento dei servizi e della qualità della vita (Ufficio di Statistica del Comune di Roma 2016).

${ }^{29}$ Un altro campo di operatività dei gruppi criminali è l'economia del mare, già profondamente intrisa di illegalità diffusa, che pure ha effetti sull'abusivismo del demanio balneare. Nello spazio qui concesso non è possibile entrare in questo campo; rinvio l'analisi a Martone (2018).

${ }^{30}$ L'uso della propria malattia contagiosa non si limita al caso in questione, ma anzi vi ricorre sistematicamente per intimidire chi è in ritardo nei pagamenti o gli inquilini degli alloggi popolari da espellere.

${ }^{31}$ Lo stato di abbandono dell'edilizia residenziale pubblica prosegue tuttora, così come le sofferenze contabili su affitti e bollette e i contenziosi amministrativi. Un dato rilevato dal «Laboratorio di Urbanistica» nel 2016 dice che su 4.172 alloggi di edilizia residenziale, ben 1.720 sono condotti in affitto passivo (di cui 1.042 nelle «palazzine Armellini» a Nuova Ostia) cui si affiancano altri 65 beni a uso non residenziale, 53 dei quali in passivo (De Jesus 2016).
} 
Nel mese di agosto [...] io ed il mio compagno abbiamo abbandonato la mansarda in Via Vasco de Gama [...] che è stata occupata da altre persone dopo molto tempo ed abbiamo occupato abusivamente una casa popolare sita in Via Baffigo [...]. Mia suocera ha invece occupato l'appartamento a fianco [...]. Approfittando del fatto che mia suocera si era allontanata dall'appartamento di Via Baffigo per andare a prelevare alcuni effetti dalla mansarda di Via Vasco de Gama, [un affiliato al clan rivale, ndr] dopo avermi sottratto le chiavi di casa di mia suocera entrava all'interno dell'appartamento e se ne impossessava buttando fuori tutti gli effetti di mia suocera. Ricordo che lui stesso disse che da quel momento in poi quella casa sarebbe stata la sua e che se avessimo avuto qualcosa da ridire sarebbe stato peggio per noi [...]. Nel mese di novembre, approfittando che da diverso tempo [...] non si faceva vedere, ci siamo ripresi l'appartamento e mia suocera è tornata a viverci (Tribunale di Roma 2015: 30).

Le occupazioni servono anche a costruire consenso: gli alloggi vengono "assegnati” alle famiglie di affiliati e non, così come negli immobili si fanno iniziative e attività aperte al pubblico, indispensabili in un'area che, come detto, versa in condizioni di disagio diffuso ${ }^{32}$. Il caso della Palestra Femus in Via Forni è emblematico in tal senso. Si tratta di una palestra installata in un immobile di proprietà del Comune di Roma, fino al 2015 gestito da membri di un nucleo familiare assai esteso, composto da parenti e affini di origine sinti e residenti nel litorale da almeno tre decenni, originariamente insediatisi in forma non stanziale (Int. parroco). Un abitante dell'area situa l'esperienza dell'occupazione abusiva della Femus nel più ampio contesto sociale:

Lì è piazza Gasparri, c’è molto disagio sociale. [...] Anche se la palestra era abusiva, era un'occupazione di un locale comunale e non pagavano l'affitto da molto tempo. Però posso dirti ... non per giustificarli eh ... ma [...] non sono i soli che occupano abusivamente dei locali a Nuova Ostia. In tutta questa situazione degli affitti, degli appartamenti abusivamente occupati, poi dentro ci finisce anche criminalità. Cioè, c'è qualcuno che dice: “mo' me ce butto pure io". Loro lo organizzano e lo fanno diventa' come un'assicurazione, un welfare dell'illegalità, delle adiacenze [...]. Qui la distribuzione delle case serve anche per avere consenso sociale. Devo dire che questi ragionamenti che stiamo facendo me li ha fatti anche una mia amica. Lei diceva: "Si però questi facevano cose buone. Nella palestra [...] facevano cose buone". La questione vera riguarda il connubio tra cose buone e legalità. Detto tra noi, un conto è che una parrocchia occupa abusivamente una roba e ci fa 'n'attività ... un conto è che il sistema [criminale] occupa un posto e ci fa una palestra. È una questione di valori. Cioè il creare socialità va inserito in un sistema di valori che si veicolano. Che gli può dire [...] ai bambini, rispetto all'attività che fa suo padre? (Int. referente associazione civica) ${ }^{33}$.

L'intervistato individua due pratiche socio-spaziali che condividono la stessa «grammatica del mondo» ${ }^{34}$, ampi margini di informalità che sfumano i confini tra la «prassi» e la «norma», ponendo le basi per forme di autorganizzazione locale e - a un tempo - per la genesi della criminalità autoctona. Quando le solidarietà che accomunavano gli abitanti degli anni Sessanta e Settanta si affievoliscono, la distanza è gradualmente mutata in indifferenza e sfiducia interpersonale, oltre che istituzionale. In queste circostanze la persistenza di sacche di povertà spazialmente circoscritta facilita la genesi di una certa regolazione violenta che proprio nelle ambivalenze dell'abitare informale trova opportunità di regolazione sociale. Profittando della medesima informalità ai margini, i gruppi criminali presidiano il territorio, assumono competenze di regolazione sociale (dirimere conflitti, garantire sicurezza) ed economica (distribuire alloggi e prebende), inducono «processi di quotidianizzazione della violenza» (Massari 2015: 237), in cui la carenza della regolazione istituzionale configura strutture di opportunità per la sopraffazione mafiosa che in alcuni casi rimpiazza quella dello Stato. Questo non vuol dire che la concentrazione dello svantaggio nelle

\footnotetext{
${ }^{32}$ Tra le varie spiccano le iniziative organizzate unitamente alla sezione ostiense di Casapound, radicatasi nel tempo proprio nella zona di Nuova Ostia, anche promuovendo manifestazioni di piazza contro lo sgombero di palazzine abusivamente occupate. Nelle elezioni municipali del 2017 Casapound - con la lista Fascisti del terzo millennio - ottiene un ragguardevole consenso, superiore al 9\%, con punte di quasi 20 punti proprio nelle zone più problematiche di Nuova Ostia e ad Acilia. Da sottolineare quanto tale suffragio si accompagni a una elevatissima astensione (affluenza al 36,15\%), sintomo anch'essa di una certa forma di radicalizzazione e di protesta politica.

${ }^{33}$ In effetti, sulla medesima via Forni in cui sorgeva la Palestra Femus, nello stesso maggio 2015 veniva alla luce che persino la locale sede del Partito Democratico occupava abusivamente un immobile.

${ }^{34}$ Discutendo dei quartieri in cui si radica la criminalità, Isaia Sales scrive che alla base del riconoscimento sociale del potere territoriale può esserci una «grammatica del mondo» condivisa all'interno di un ambiente «che sente il comportamento mafioso non estraneo e non esterno ai suoi codici» (Sales 2015: 207). Una grammatica che non presume la sistematica legittimazione del potere criminale, ma non esclude l'esistenza di un vocabolario condiviso dove tale potere è quotidianamente praticato.
} 
periferie possa essere utilizzata per sostenere una correlazione diretta e sistematica tra fragilità sociale e genesi della criminalità urbana.

\section{MARGINALITÀ URBANE TRA QUESTIONE SOCIALE E QUESTIONE CRIMINALE: RIFLESSIONI CONCLUSIVE}

Riflettendo sulle economie criminali in ambiente metropolitano, l'antropologo Michel Peraldi, noto per i numerosi studi sulle reti del commercio informale nel Mediterraneo, più di dieci anni fa sottolineava che:

sembra ormai difficile ragionare come se queste economie appartenessero a una "nicchia" sociale autonoma, esterna allo spazio-tempo della città [...]. Le scienze sociali esitano tra la tentazione di sottovalutare l'impatto di queste economie nei contesti urbani circoscrivendole a determinati mondi etnici e sociali, con il rischio di una etnicizzazione criminalizzante dei mondi in questione, e una sopravvalutazione paranoica del controllo e della potenza degli imprenditori e delle organizzazioni dell'economia criminale (Peraldi 2009: 540).

Rispetto al caso romano, nell'interpretare i fenomeni appena ricostruiti, questa impasse mette di fronte due contrapposte letture prevalenti: da un lato, chi sostiene che l'etichetta "mafia" vada intesa in un più ampio processo di blaming che delega le funzioni sgradite alle periferie, facendone capro espiatorio della crisi del centro (cfr. ad esempio Portelli 2018). Dall'altro lato, chi si sofferma sul processo di naming e, al fine di contrastare «una lettura fortemente negazionista o riduzionista» (Benincasa 2021: 74), spinge per l'applicazione - che sia penale o «morale» - dell'etichetta "mafia" ai gruppi criminali romani come questione prioritaria nei problemi della città. La prima impostazione, talvolta apologetica di una certa spontaneità dei margini, ha il pregio di individuare il problema dell'uso politico della sicurezza, ma in alcuni passaggi rischia di ridimensionare il potere criminale e l'uso diffuso della violenza organizzata nei contesti periferici, che pure produce altrettanta disuguaglianza ${ }^{35}$. La seconda ha il merito di porre l'attenzione su fenomeni che in letteratura - fino ad anni recentissimi - sembravano relegati nella «"nicchia" sociale autonoma» di cui parla Michel Peraldi. Questa seconda impostazione è tuttavia più problematica, perché tende a adottare acriticamente il punto di vista del contrasto penale e ad alimentare arrischiate letture mafiocentriche, proprie di un'idea di mafia come oggetto monolitico e totalizzante. Modelli causali in cui le mafie - intese come associazioni, logiche d'azione, strategie imprenditoriali, reti d'affari - sono variabile indipendente (Sciarrone 2009; Sciarrone 2019c), producendo spiegazioni tautologiche (la criminalità è indistinguibile dal suo contesto e il contesto è criminale) e suggerendo strategie di contrasto prive di visione politica. $\mathrm{O}$ meglio, tale impostazione depoliticizza il contrasto alle disuguaglianze urbane costringendole nelle maglie della giustizia penale, così affidando alle istituzioni di contrasto tanto il presidio - militare e tecnologico - del territorio, quanto il potere di definire diagnosi e terapie per i contesti marginali ${ }^{36}$. Adotto vocaboli prestati dal campo sanitario perché, semplificando, nella costruzione dell'indagine giudiziaria la criminalità viene vista come un agente patogeno che infetta un corpo sociale altrimenti sano, e basterebbe espellerla per curare la città. Se si riduce il dibattito politico al dibattito giuridico-giudiziario, si assume una griglia interpretativa che disperde i motivi sociali, economici e politici che presiedono al regime della disuguaglianza e al disagio abitativo che ne è l'implicazione più propriamente urbana.

È quanto accade a Ostia. Qui l'efficacia della repressione penale - che ha riconosciuto la mafiosità dei gruppi criminali locali - si riverbera sul dibattito pubblico, che continua a contrapporre posizioni allarmiste ( Ostia

\footnotetext{
${ }^{35}$ Riflessione simile è in Ciconte (2021: 221).

${ }^{36}$ Ad esempio, analizzando l'ownership nei processi di definizione dei problemi pubblici a Roma, Elena Ciccarello individua un processo di «delega » della politica alle istituzioni di contrasto - magistratura in primis - rispetto ad altri saperi esperti (Ciccarello 2021). Va rimarcato che in questo scenario non è sempre la magistratura a occupare strategicamente il discorso pubblico, quanto appunto - la politica a lasciare campo vuoto. Sul punto, gli stessi magistrati da me intervistati sottolineano la parzialità della prospettiva giudiziaria - indicata come «una versione patologica della realtà » - per la comprensione della complessità sociale.
} 
come Corleone» ${ }^{37}$ ) a tesi negazioniste («qui non si paga il pizzo» ${ }^{38}$ ), schiacciando il dibattito politico sulla diatriba «mafia/non mafia» e sfociando in inevitabili processi di semplificazione e di etichettamento. Anche nel quadro di tali esasperazioni possono leggersi le manifestazioni e le prese di posizione promosse da fazioni politiche, associazioni di categoria e forze sociali di diversi schieramenti, durante l'intensa campagna elettorale dell'autunno 2017, vinta dalla candidata del M5S, Giuliana Di Pillo. Una campagna che ha posto al centro del dibattito proprio legalità e sicurezza (Lucianetti 2018), temi trasversali ai programmi delle principali liste, compresi i Fascisti del terzo millennio che, come detto, in quella tornata ottengono un consenso ragguardevole. Nell'area di Nuova Ostia, priva di rappresentanza e staccata da un orizzonte di rivendicazione collettiva, si alimenta un cortocircuito tra radicalizzazione, opzioni neopopuliste e xenofobe, contese spaziali e ulteriori spinte emergenziali e repressive.

Spostare il fuoco dalla mera sorveglianza di quartieri pericolosi alla complessità dei margini risulta non solo più proficuo da un punto di vista analitico, ma anche più efficace nelle proposte politiche che mirano ad alimentare attivandole dall'interno - reti e forze sociali di cui il quartiere già dispone, affrontando le ragioni di quel disagio socio-spaziale, ricostruendo le reti e ri-occupando i luoghi capaci di tradurlo in istanze collettive e di arginare i circuiti di regolazione violenta.

\section{BIBLIOGRAFIA}

Agier M. (2014, cur.), Un monde de camps, Parigi: La Découverte.

Allen J. (2006), Welfare Regimes, Welfare Systems and Housing in Southern Europe, in «International Journal of Housing Policy», 6: 251-277.

Angeli F. (2020), Roma. 2000 anni di corruzione, Roma: Baldini \& Castoldi.

Battistelli F., Lucianetti L.F. (2010), La sicurezza tra politics e policy, in A. Pajno, La sicurezza urbana, Rimini: Maggioli.

Benigno F. (2015), La mala setta. Alle origini di mafia e camorra. 1859-1878, Torino: Einaudi.

Benincasa G. (2021), Mala Capitale. Cosa resta della più grande inchiesta contro la criminalità capitolina?, Roma: Castelvecchi.

Benini R., De Nardis P. (2013), Capitale senza capitale. Roma e il declino d'Italia, Roma: Donzelli.

Bertuzzi N., Caciagli C., Caruso L. (2019), Popolo chi? Classi popolari, periferie e politica in Italia, Roma: Ediesse.

Bulfon F. (2019), Casamonica, la storia segreta, Milano: Rizzoli.

Caciagli C. (2018), La casa fra rivendicazioni dei movimenti e approccio istituzionale, in E. D’Albergo e D. De Leo, Politiche urbane per Roma. Le sfide di una Capitale debole, Roma: SUP.

Capaldo G. (2013), Roma mafiosa. Cronache dell'assalto criminale allo Stato, Roma: Fazi.

Carminucci C., Casucci S., Frisch G. (2014), Roma, una città metropolitana in crescita e trasformazione, in «Meridiana $\gg, 80: 77-103$.

Cassetti R. (2008), Roma e Lazio. La formazione di una regione urbana, Roma: Gangemi.

Casucci S., Leon A. (2014), Cambiamenti in atto nelle città metropolitane del nostro Paese, in «Meridiana», 80: $19-40$.

Cellamare C. (2019), Città fai-da-te. Tra antagonismo e cittadinanza. Storie di autorganizzazione urbana, Roma: Donzelli.

Cellamare C. (2016, cur.), Fuori Raccordo. Abitare l'altra Roma, Roma: Donzelli.

Cellamare C. (2008), Pratiche e politiche urbane. Spunti di riflessione a partire da una ricerca in corso, in G. Imbesi, R. Lenci e M. Sennato, Urban practice and policy. Reflections from some ongoing research, Roma: Gangemi.

Chiodelli F., Coppola A., Belotti E., Berruti G., Clough Marinaro I., Curci F., Zanfi F. (2021), The production of informal space: A critical atlas of housing informalities in Italy between public institutions and political strategies, in «Progress in Planning», 149, https://doi.org/10.1016/j.progress.2020.100495.

\footnotetext{
${ }^{37}$ Saviano R., “Ostia è diventata come Corleone e Scampia, territorio dei clan”, in Repubblica TV, 8 novembre 2017.

${ }^{38}$ Carli A., "Ostia, le associazioni dei commercianti: «L'usura strozza le nostre imprese»”, in Sole24Ore, 27 novembre 2017.
} 
Ciccarello E. (2021), La mafia come problema pubblico. Il ruolo del potere giudiziario nella scoperta delle "mafie di Ostia", in «Studi sulla questione criminale», 2, maggio-agosto: 35-57.

Ciccarello E. (2016), La posta in gioco di Mafia Capitale. Nuove mafie e interpretazione del reato di associazione mafiosa, in «Meridiana», 87: 65-89.

Ciconte E. (2021), L'assedio. Storia della criminalità a Roma da Porta Pia a Mafia Capitale, Roma: Carocci.

Cittadini del mondo (2018), Palazzo Selam: la città invisibile, Roma.

Coppola A. (2018), La politica del condono edilizio: dimensioni, governance e processo produttivo, in E. D'Albergo e D. De Leo (cur.), Politiche urbane per Roma. Le sfide di una Capitale debole, Roma: SUP.

Coppola A. (2016), Roma: la metropolizzazione parassitaria e i suoi modi informali, in C. Cellamare, Fuori Raccordo. Abitare l'altra Roma, Roma: Donzelli.

Creti L. (2008), Il lido di Ostia, Roma: Istituto Poligrafico dello Stato.

D’Albergo E., Moini G. (2015), Il regime dell'Urbe. Politica, economia e potere a Roma, Roma: Carocci.

Davis M. (2005), Planet of Slums, Londra: Verso.

De Jesus P.F. (2016), Urbanistica sociale per Nuova Ostia: il pasticcio del Comune di Roma, in «Laboratorio di Urbanistica », http://www.labur.eu/public/blog/2016/02/15/urbanistica-sociale-per-nuova-ostia-il-pasticcio-delcomune-di-roma/.

De Leo D. (2016), Mafie \& urbanistica. Azioni e responsabilità dei pianificatori nei territori contesi alle organizzazioni criminali, Milano: Franco Angeli.

Dna (2012), Relazione annuale sulle attività svolte nel periodo $1^{\circ}$ luglio 2011 - 30 giugno 2012, Roma.

Dna (2016), Relazione annuale sulle attività svolte nel periodo $1^{\circ}$ luglio 2014 - 30 giugno 2015, Roma.

Farruggia F., Ricotta G. (2010), Sicurezza urbana e periferie. Due studi di caso a Roma, Roma: Aracne.

Fregolent L. (2008, cur.), Periferia, periferie, Roma: Aracne.

Grazioli M., Caciagli C. (2017), The right to (stay put in): The case of Porto Fluviale in Roma, in «Urbanistica Tre i Quaderni», 13, 5: 79-85.

Hassel A., Palier B. (2020), Growth \& Welfare in Advanced Capitalist Economies, Oxford: Oxford University Press.

Insolera I. (2011), Roma moderna. Da Napoleone I al XXI secolo, Torino: Einaudi.

Laino G. (2020, cur.), Politiche urbane per le periferie, Bologna: il Mulino.

Lelo K., Monni S., Tomassi F. (2019), Le Mappe della disuguaglianza. Una geografia sociale metropolitana, Roma: Donzelli.

Lucianetti L.F. (2018), Le issues della campagna elettorale nel Municipio X: tra elusione, convergenza, competizione, in E. D’Albergo e D. De Leo, Politiche urbane per Roma. Le sfide di una Capitale debole, Roma: SUP.

Martone V. (2018), Il Lungomuro di Roma. Governance e area grigia del litorale ostiense, in P. De Salvo e A. Pochini, La città in trasformazione. Flussi, ritmi urbani e politiche, Roma: Aracne.

Martone V. (2017), Le mafie di mezzo. Mercati e reti criminali a Roma e nel Lazio, Roma: Donzelli.

Massari M. (2015), Per una fenomenologia della violenza mafiosa, in M. Santoro, Riconoscere le mafie. Cosa sono, come funzionano, come si muovono, Bologna: il Mulino.

Meccia A. (2017), Lazio, la periferia di Roma, in M. Carta e D. Chirico, Under. Giovani, Mafie, Periferie, Roma: Giulio Perrone Editore.

Norris P., Inglehart R. (2019), Cultural Backlash. Trump, Brexit, and Authoritarian Populism, Cambridge: Cambridge University Press.

Orsatti P. (2015), Roma brucia. Mafia, corruzione e degrado. Il sistema di potere che stritola Roma, Reggio Emilia: Imprimatur.

Palumbo A., Scott A. (2019), Remaking Market Society. A Critique of Social Theory and Political Economy in Neoliberal Times, Londra-New York: Routledge.

Paone S. (2012), La città del disordine. Marginalità, sorveglianza, controllo, Pisa: ETS.

Paone S., Petrillo A., Chiodelli F. (2017), Governare l'ingovernabile. Politiche degli slum nel XXI secolo, Pisa: ETS.

Petrillo A. (2013), Peripherein. Pensare diversamente la periferia, Milano: Franco Angeli. 
Peraldi M. (2009), Economie criminali e mondo degli affari a Tangeri, in G. Gribaudi, Traffici criminali. Camorra, mafie e reti internazionali dell'illegalità, Torino: Bollati Boringhieri.

Pignatone G.,Prestipino M. (2019), Modelli criminali. Mafie di ieri e di oggi, Roma-Bari: Laterza.

Pitch T. (2013), Contro il decoro. L'uso politico della pubblica decenza, Roma-Bari: Laterza.

Portelli A. (2018), Ostia, mafia capitale e il rito del capro espiatorio, in L. Rossomando, Lo stato delle città, Napoli: Napoli Monitor.

Puccini E. (2018), Verso una politica della casa: aspetti e questioni dell'emergenza abitativa romana, in A. Coppola e G. Punziano (cur.), Roma in transizione. Governo, strategie, metabolismi e quadri di vita di una metropoli, Vol. 1, Roma-Milano: Planum.

Puccini E. (2016), Verso una politica per la casa, dall'emergenza abitativa romana ad un nuovo modello nazionale, Roma: Ediesse.

Rodriguez-Pose A. (2017), The revenge of the places that don't matter (and what to do about it), in «Cambridge Journal of Regions, Economy and Society», 11, 1: 189-209.

Sabella A. (2016), Capitale infetta, Milano: Rizzoli.

Sales I. (2015), Storia dell'Italia mafiosa, Soveria Mannelli: Rubbettino.

Savatteri G., Grignetti F. (2015), Mafia Capitale. L'atto di accusa della Procura di Roma, Melampo, Milano.

Sciarrone R. (2009), Mafie vecchie, mafie nuove. Radicamento ed espansione, Roma: Donzelli.

Sciarrone R. (2019a), Forms of Capital and Mafia Violence, in M. Massari e V. Martone, Mafia Violence. Political, Symbolic, and Economic Forms of Violence in Camorra Clans, Londra-New York: Routledge.

Sciarrone R. (2019b, cur.), Mafie del nord. Strategie criminali e contesti locali, Roma: Donzelli.

Sciarrone R. (2019c), Tra Sud e Nord. Le mafie nelle aree non tradizionali, in R. Sciarrone, Mafie del nord. Strategie criminali e contesti locali, Roma: Donzelli.

Selmini R. (2020), Dalla sicurezza urbana al controllo del dissenso politico, Roma: Carocci.

Touraine A. (1991), Face à l'exclusion, in «Esprit», 169, 2: 7-13.

Tribunale di Roma (2015), Ordinanza applicativa della custodia cautelare in carcere nei confronti di Spada Ottavio e altri.

Tribunale di Roma (2018), Ordinanza di custodia cautelare in carcere nei confronti di Spada Carmine e altri.

Trigilia C. (2020), Capitalismi e democrazie. Si possono conciliare crescita e uguaglianza?, Bologna: il Mulino.

Trocchia N. (2019), Casamonica. Viaggio nel mondo parallelo del clan che ha conquistato Roma, Roma: Utet.

Ufficio di Statistica del Comune di Roma (2016), Popolazione maschile e femminile iscritta in anagrafe per zone urbanistiche.

Vereni P. (2015), Addomesticare il welfare dal basso. Prospettive e paradossi delle occupazioni abitative romane, in «Meridiana», 83: 147-169.

Violante A., Vicari P. (2018), Ancora un'eccezione? La crisi fiscale della Capitale tra Neoliberismo e presunto declino, in A. Coppola e G. Punziano, Roma in transizione. Governo, strategie, metabolismi e quadri di vita di una metropoli, Vol. 1, Roma-Milano: Planum.

Visconti C. (2015), A Roma una mafia c’è, e si vede..., in «Diritto Penale Contemporaneo», www.penalecontemporaneo.it.

Wacquant L. (2008), Urban outcasts: A comparative sociology of advanced marginality, Cambridge: Polity Press.

Wuthnow R. (2018), The Left Behind. Decline and Rage in Rural America, Princeton: Princeton University Press. 